\begin{tabular}{|c|c|c|c|}
\hline $\begin{array}{l}\text { Submission: } 25 / \mathrm{Jan} / 2021 \text {; } \\
\text { Camera ready: } 15 / \mathrm{Jul} / 2021 \text {; }\end{array}$ & $\begin{array}{l}1^{\text {st }} \text { round notif.: } 05 / \mathrm{May} / 2021 \text {; } \\
\text { Edition review: } 22 / \mathrm{Jul} / 2021 \text {; }\end{array}$ & $\begin{array}{l}\text { New version: 24/May/2021; } \\
\text { Available online: 06/Aug/2021; }\end{array}$ & $\begin{array}{l}2^{\text {nd }} \text { round notif:: } 30 / \mathrm{Jun} / 2021 \text {; } \\
\text { Published: } 06 / \mathrm{Aug} / 2021 ;\end{array}$ \\
\hline
\end{tabular}

\title{
Perspectivas mundiais sobre a realidade aumentada nos anos iniciais da educação básica
}

\section{Title: Global perspectives on augmented reality in the early years of basic education}

\author{
Janaina Schlickmann Klettemberg \\ Universidade Federal de Santa Maria- \\ UFSM \\ janaina.klettemberg@acad.ufsm.br
}

\author{
Romero Tori \\ Universidade de São Paulo - USP \\ tori@usp.br
}

\author{
Camila Maldonado Huanca \\ Universidade de São Paulo - USP \\ camila.huanca@usp.br
}

\section{Resumo}

A realidade aumentada possibilita que o usuário interaja com objetos reais e virtuais simultaneamente em um ambiente do mundo real. Seus recursos podem ser utilizados para auxiliar o processo educacional devido ao caráter inovador, interativo e pela capacidade de experimentação e contextualização proporcionada. O objetivo desta revisão integrativa foi investigar as perspectivas mundiais sobre o uso de realidade aumentada no ensino fundamental (anos iniciais) da educação básica. Os 55 artigos incluídos estudaram a realidade aumentada utilizada por crianças em idade escolar entre 6 e 11 anos. Constatou-se que a realidade aumentada pode melhorar os processos de ensino e aprendizagem, possibilitando melhora da aquisição do conhecimento, motivação, interação e engajamento.

Palavras-Chave: Realidade Aumentada; educação; Ensino Fundamental; educação básica; informática na educação.

\begin{abstract}
Augmented reality allows simultaneous interaction of real and virtual objects in a real world environment. Its resources can be used to assist the educational process due to its innovative, interactive character and the ability to experiment and provide context. The aim of this integrative review was to investigate the global perspectives on the use of augmented reality in the early years of elementary education. The 55 articles included studied the augmented reality used by schoolchildren aged 6 to 11 years. It was found that augmented reality can improve the teaching and learning processes, allowing for an improvement in the acquisition of knowledge, motivation, interaction and engagement.
\end{abstract}

Keywords: Augmented Reality; education; elementary education; K12; computers in education.

Cite as: Klettemberg, J. S., Tori, R., \& Huanca, C. M. (2021). Perspectivas mundiais sobre a realidade aumentada nos anos iniciais da educação básica. Revista Brasileira de Informática na Educação, 29, 827-845. DOI: 


\section{Introdução}

A educação é uma teia complexa de processos que se estabelecem na relação dialética entre sujeitos que se relacionam com outros e com o mundo. Nessa complexidade, a busca por recursos e metodologias pautadas no protagonismo do educando são essenciais para melhorar a qualidade do ensino no mundo.

Os recursos tecnológicos têm demonstrado grande eficácia nos processos de ensino e aprendizagem. Conforme Tajra (2019), a tecnologia educacional pretende transformar o processo de aprendizagem em algo mais dinâmico e interativo, provocando mudanças sociais, culturais e econômicas, e também reestruturando a educação e o papel do professor.

Entre as mais recentes tecnologias educacionais destaca-se a Realidade Aumentada (RA), que abre muitas possibilidades de inovação na aprendizagem ao trazer conteúdos virtuais para o espaço físico onde o aluno se encontra. Mas por ser uma mídia relativamente nova ainda possui muitos desafios de pesquisa e desenvolvimento (Tori, 2017).

Conforme Azuma et al. (2001), a RA é definida como um sistema capaz de complementar o mundo real com objetos virtuais gerados por computador que parecem coexistir no mesmo espaço. Quando o usuário está em um ambiente real e consegue interagir com elementos virtuais devidamente registrados tridimensionalmente no espaço físico real, concebemos que está ocorrendo a RA. Assim, a RA combina objetos reais e virtuais no ambiente real; possui interatividade e é aplicada aos sentidos humanos. Essas características são fundamentais para a caracterização de um sistema ou aplicação de RA (Azuma et al., 2001). Pode-se dizer, portanto, que a RA complementa o mundo real adicionando informações sensoriais, tais como sons, imagens, vídeos, objetos e enriquecendo a experiência do usuário (Kirner \& Siscoutto, 2007).

Desde o século XVII, já existia a ideia de aumentar a realidade com recursos, como espelhos e lentes. Mas foi somente em 1968 que houve a criação do primeiro protótipo de dispositivo que permitia juntar imagens geradas por um computador no formato tridimensional a imagens reais (Tori \& Hounsell, 2018). Desde a década de 1990, a RA tem se disseminado e recebido investimentos de diversas áreas: entretenimento, engenharias, saúde e educacionais.

Algumas características observadas na RA contribuem para a melhora na aprendizagem dos alunos, como: interatividade, experimentação, ambiente seguro, contextualização de conceitos abstratos, percepção, motivação e engajamento (Tori \& Hounsell, 2018).

Com a RA fica fácil promover a aprendizagem ativa e, por esse motivo, há sucesso quando é possibilitado ao aluno que ele experimente e interaja com o objeto do conhecimento (Bacich \& Morin, 2018). Através da RA, o aluno pode experimentar viagens espaciais, fazer tentativas seguras de experimentos científicos, bem como visualizar e manusear objetos não fornecidos com facilidade nas escolas. A RA amplia as possibilidades de visualização, interação e imersão dos conteúdos escolares.

Além disso, diversos aspectos emocionais e psicológicos são atendidos com o uso da RA. Utilizando a RA como recurso pedagógico, a interatividade aumenta e, consequentemente, a motivação e o engajamento. Sendo assim, a RA apresenta-se como um excelente recurso pedagógico inovador, contribuindo no processo de ensino e aprendizagem, atendendo e resolvendo conflitos emocionais encontrados no espaço educativo, como engajamento e motivação, e consequentemente favorecendo a aprendizagem dos educandos.

A revisão relatada neste artigo teve por objetivo investigar o uso da RA no ensino fundamental (anos iniciais) da educação básica no que tange aos aspectos emocionais, tecnológicos e a aquisição do conhecimento. O enfoque foi nas práticas pedagógicas exitosas que fizeram uso desta tecnologia no Brasil e no mundo e que foram analisadas cientificamente e tiveram os resultados publicados. Sabe-se que há uma grande quantidade de ferramentas de RA, 
como por exemplo o Augie AR lab, com potencial educacional, mas o foco dessa pesquisa esteve centrado na análise de publicações testadas com rigor metodológico e devidamente públicas à comunidade científica.

Esse estudo originou-se na inexistência de trabalhos correlatos que investigaram o uso da RA nos anos iniciais da educação básica. Há pesquisas que abordam a RA na educação de forma abrangente (Sirakaya \& Alsancak, 2018) (Bacca Acosta et al., 2014) e na educação especial (Izaguirre \& Collazos, 2020) (Khowaja et al., 2020). Há apenas um estudo que aborda o ensino de Matemática, através de jogos com o uso de RA, no ensino fundamental e médio (Pellas et al., 2019). Contudo, não há revisões sistemáticas dedicadas aos anos iniciais da educação básica, sendo este um fator motivacional desse estudo.

Esta pesquisa justifica-se pela busca, em referências bibliográficas, de cenários que utilizam a RA nos anos iniciais. No ensino fundamental (anos iniciais) os alunos passam por diversas mudanças, tanto nos aspectos cognitivos como também nos emocionais, sociais e físicos. Diante de tantas mudanças, é preciso escolher recursos pedagógicos engrandecedores que facilitem todos os processos de mudança e principalmente o processo de ensino e aprendizagem. É nos anos iniciais que os alunos passam pelo período de transição da educação infantil aos anos finais e a articulação entre esses períodos deve possibilitar experiências vívidas. A RA tem a potencialidade de oferecer a integração e a experimentação tão necessárias a essa etapa escolar. Por isso, ao abordar os cenários de usos da RA, a pesquisa pretende criar uma fonte de conhecimento extremamente válida que faz comparativos dos aspectos emocionais, tecnológicos e de aquisição do conhecimento. Abordar as informações demográficas permite uma análise global das ações que estão sendo feitas mundialmente e a possibilidade de traçar novos rumos compreendendo a assertividade em determinados locais.

Para alcançar os objetivos da pesquisa aqui relatada foi utilizado o método de revisão integrativa da literatura, seguindo seis etapas distintas (Mendes, Silveira, \& Galvão, 2008): elaboração da pergunta norteadora; busca ou amostragem na literatura; coleta de dados; análise crítica dos estudos incluídos; discussão dos resultados e apresentação da revisão integrativa (Ganong, 1987).

Com relação às bases de pesquisa, essas foram delimitadas aos Periódicos Capes $^{1}$, Congresso Brasileiro de Informática na Educação $(\mathrm{CBIE})^{2}$, Simpósio Brasileiro de Informática na Educação $(\mathrm{SBIE})^{3}$, ERIC - Institute of Education Sciences ${ }^{4}$ IEEE $^{5}$ e SCOPUS $^{6}$.

É importante salientar que o ensino fundamental (anos iniciais) da educação básica difere em nomenclatura nos diversos países. Contudo, é consenso que os anos iniciais correspondem à faixa etária dos educandos que vai dos seis aos onze anos de idade, seguindo principalmente a Classificação Internacional Normalizada da Educação (ISCED).

Em virtude da variedade de nomenclaturas que delimita o ensino fundamental (anos iniciais) da educação básica em diversos países, e buscando uma grande captação de trabalhos publicados, foram utilizadas as palavras-chave "realidade aumentada" e "educação", e suas respectivas formas em inglês, combinadas a descritores diversos, sendo eles: educação básica, anos/séries iniciais, ensino fundamental, augmented reality, elementary school, elementary education, primary school, primary education e grade 1, 2, 3, 4 e 5.

\footnotetext{
${ }^{1} \mathrm{https}: / /$ www.periodicos.capes.gov.br

${ }^{2}$ https://www.br-ie.org/pub/index.php/wcbie/search

${ }^{3}$ https://www.br-ie.org/pub/index.php/sbie/index

${ }^{4}$ https://eric.ed.gov/

${ }^{5} \mathrm{https}: / /$ www.ieee.org/

${ }^{6} \mathrm{https}: / /$ www.scopus.com/
} 
Foram incluídos artigos com texto integral disponíveis de forma eletrônica e gratuito, que continham alguma das palavras-chave mencionadas na pesquisa no título e/ou resumo, e compatibilidade com os objetivos desta revisão integrativa. Os artigos excluídos foram aqueles que: não corresponderam ao nível de ensino em análise; abordaram alguma das modalidades específicas da educação (Educação Escolar Indígena; Educação Especial; Educação do Campo; Educação Escolar Quilombola; Educação de Jovens e Adultos e Educação Profissional); ou eram estudos secundários (revisão teórica ou mapeamento). O processo de busca ocorreu nos meses de junho e julho de 2020.

\section{Resultados}

De início, fazendo busca livre com os termos descritos anteriormente, foram encontrados 445 artigos, dos quais, utilizando os critérios de inclusão e exclusão, foram selecionados 94 artigos nas seis bases de dados relatadas. Seguindo os protocolos da revisão integrativa, foi realizada a análise crítica dos estudos chegando ao total de 55 artigos no portfólio final.

A Figura 2.1 detalha o processo de seleção documental.

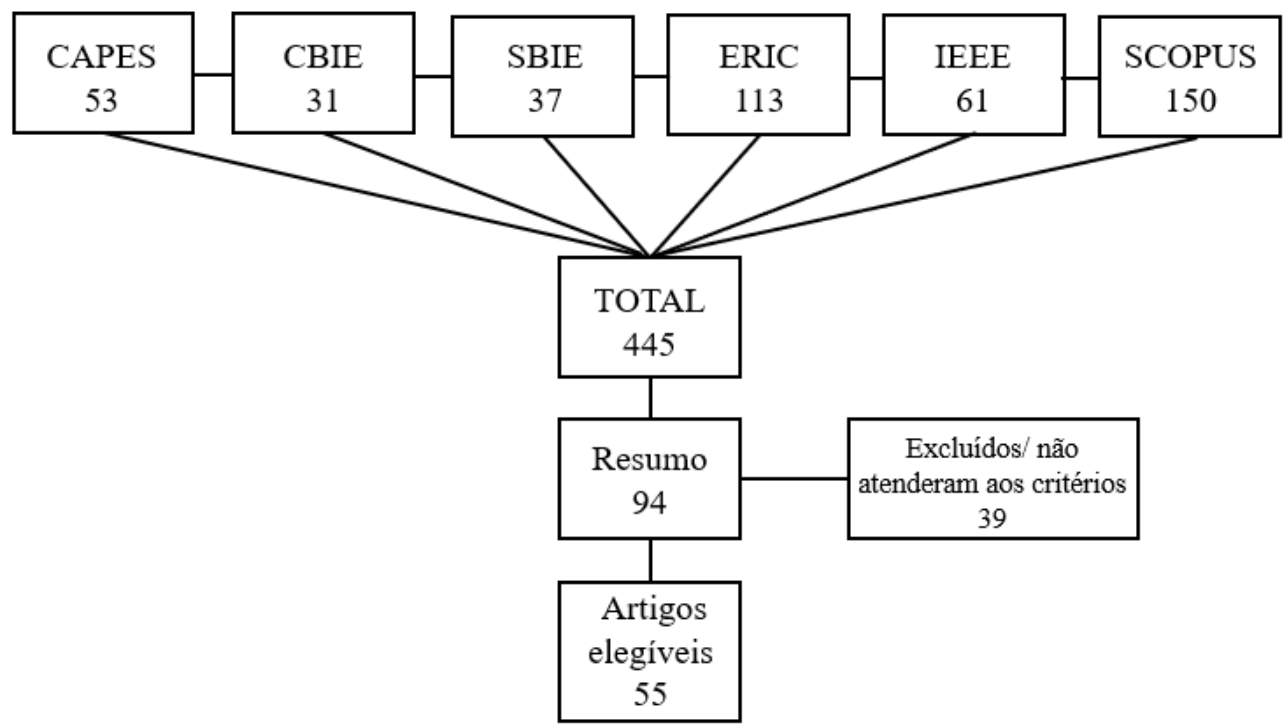

Figura 2.1. Processo de seleção documental.

Após a leitura dos artigos selecionados, foram extraídos os dados principais e agrupados por assuntos similares. A tabela 2.1 apresenta todos os artigos selecionados.

Tabela 2.1. Artigos selecionados.

\begin{tabular}{|l|l|l|}
\hline $\mathbf{N}^{\mathbf{0}}$ & Tema & Referência \\
\hline D1 & ensino de frações & (Silva et al., 2014) \\
\hline D2 & ciências sociais & (Toledo-Morales\&Sanches-Garcia, 2018) \\
\hline D3 & ensino de história & (Casado et al., 2018) \\
\hline D4 & jogo para auxílio na alfabetização & (Silva et al., 2017) \\
\hline D5 & RA móvel para apoio ao ensino de crianças & (Cavalcante, 2016) \\
\hline
\end{tabular}




\begin{tabular}{|c|c|c|}
\hline D6 & jogo online sobre classificação dos animais & (Flauzino \& Kirner, 2013) \\
\hline D7 & materiais didáticos para educação básica & (Gotardo et al., 2013) \\
\hline D8 & jogo para auxiliar no aprendizado infantil & (Oliveira \& Zorzal, 2013) \\
\hline D9 & livro interativo: Banda da Floresta & (Carneiro \& Silva, 2016) \\
\hline D10 & RA móvel no ensino de ciências naturais & (Hendajani. et al., 2019) \\
\hline D11 & vocabulário da língua inglesa & (Hsu, 2019) \\
\hline D12 & ciências naturais & (Hendajani et al., 2018) \\
\hline D13 & visão espacial & (Phon et al., 2019) \\
\hline D14 & raciocínio abstrato no aprendizado de ciências & (Syawaludin et al., 2019) \\
\hline D15 & compreensão de texto (comparação com impresso) & (Danaei et al., 2020) \\
\hline D16 & desafios e perspectivas em país em desenvolvimento & (Alalwan et al., 2020) \\
\hline D17 & geometria & (Purnama et al., 2014) \\
\hline D18 & esqueleto humano & (Kouzi et al., 2019) \\
\hline D19 & aplicações em matemática & (Radu et al., 2016) \\
\hline $\mathrm{D} 20$ & ciências & (Maijarern et al., 2018) \\
\hline D21 & jogo sobre nomes de animais em inglês & (Barreira. et al., 2012) \\
\hline D22 & ciências naturais & (Fakhrudin et al., 2017) \\
\hline D23 & design universal para aprendizagem & (Stylianidou. et al., 2020) \\
\hline D24 & RA móvel no letramento científico & (Winarni \& Purwandari, 2019) \\
\hline $\mathrm{D} 25$ & sala de aula de linguagem & (Solak \& Cakir, 2015) \\
\hline D26 & matemática básica & Lozada-Yánez et al., 2019) \\
\hline D27 & aprendizagem colaborativa (Musical Peddy-Paper) & (Gomes et al., 2014) \\
\hline D28 & impacto na aquisição e retenção de conhecimento & (Pérez-López \& Contero, 2013) \\
\hline $\mathrm{D} 29$ & influência dos tipos de jogos no interesse dos alunos & (Chen et al., 2020) \\
\hline D30 & botânica (comparação com vídeo) & (Chang et al., 2014) \\
\hline D31 & efeitos de um jogo no aprendizado e atitudes & (Hwang et al., 2016) \\
\hline D32 & efeitos de jogo puzzle na aprendizagem e motivação & $(\mathrm{Lu}$ et al., 2020) \\
\hline D33 & ensino de história baseado em jogos & (Oltman \& Hammond, 2017) \\
\hline D34 & avaliação formativa (estudo comparativo) & (Bhagat et al., 2019) \\
\hline D35 & festivais culturais chineses & (Chang et al., 2014) \\
\hline D36 & ecologia das borboletas & (Tarng \& Ou, 2012) \\
\hline D37 & vocabulário de inglês baseado em jogo & (Chen et al., 2018) \\
\hline D38 & jogo com suporte sócio-cognitivo & (Wen, 2020) \\
\hline D39 & classificação dos animais & (Savitri et al., 2019) \\
\hline $\mathrm{D} 40$ & RA móvel na aprendizagem da língua Quechua & (Camacho et al., 2019) \\
\hline
\end{tabular}




\begin{tabular}{|l|l|l|}
\hline D41 & geometria & (Rossano et al., 2020) \\
\hline D42 & sistemas digestório e circulatório & (Pérez-López et al., 2010) \\
\hline D43 & meio-ambiente & (Wickramapala, 2019) \\
\hline D44 & ciências: ciclo da água & (Bratitsis et al., 2017) \\
\hline D45 & aprendizagem colaborativa de música (Symphony-Q) & (Kusonoki et al., 2002) \\
\hline D46 & vocabulário da língua inglesa & (Chen \& Wang, 2015) \\
\hline D47 & estratégias para cálculo de volumes de cubos & (Yu et al., 2016) \\
\hline D48 & vocabulário chinês (comparação com flashcards) & (Tsai, 2018) \\
\hline D49 & geometria & (Radu et al., 2015) \\
\hline D50 & língua inglesa & (Hsu, 2017) \\
\hline D51 & matemática: jogo colaborativo MathBuilder & (Stappen et al., 2019) \\
\hline D52 & padrões de interação & (Chiang et al., 2014) \\
\hline D53 & RA móvel no letramento científico & (Wahyu et al., 2020) \\
\hline D54 & compreensão auditiva & (Del Río Guerra et al., 2020) \\
\hline D55 & redução de ansiedade em matemática & (Wangid et al., 2020) \\
\hline
\end{tabular}

A partir da análise dos artigos selecionados foi possível identificar alguns cenários mundiais sobre o uso de RA no ensino fundamental (anos iniciais) da educação básica.

A figura 2.2 apresenta os artigos catalogados pelo ano de publicação. A linha de tendência demonstra o aumento do interesse pela comunidade científica na temática.

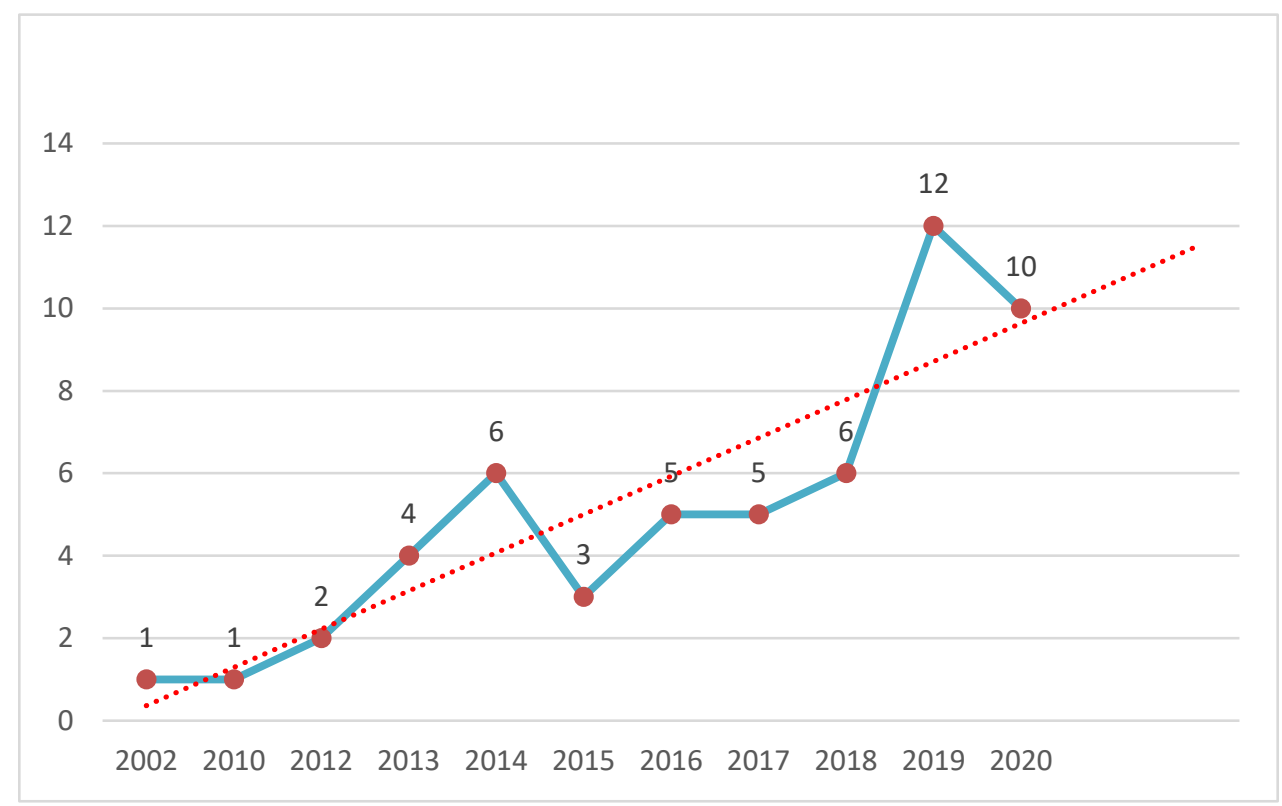

Figura 2.2. Número de artigos publicados anualmente.

O uso da RA no ensino fundamental (anos iniciais) da educação básica é globalmente difundido na perspectiva deste estudo, que totalizou a análise de 55 artigos. Destaca-se, nessa 
análise, o número de publicações em cada continente, sendo 31 publicações no continente asiático; 10 na Europa; 8 na América do Sul e 6 na América do Norte.

Os artigos foram catalogados conforme o ano escolar que abordam. Alguns recursos de RA possibilitam a aplicação em mais de um ano escolar, enquanto outros são específicos para abordagens em um determinado período. A totalidade dos artigos foi organizada na figura 2.3 possibilitando a visualização do número de recursos de RA em cada ano escolar.

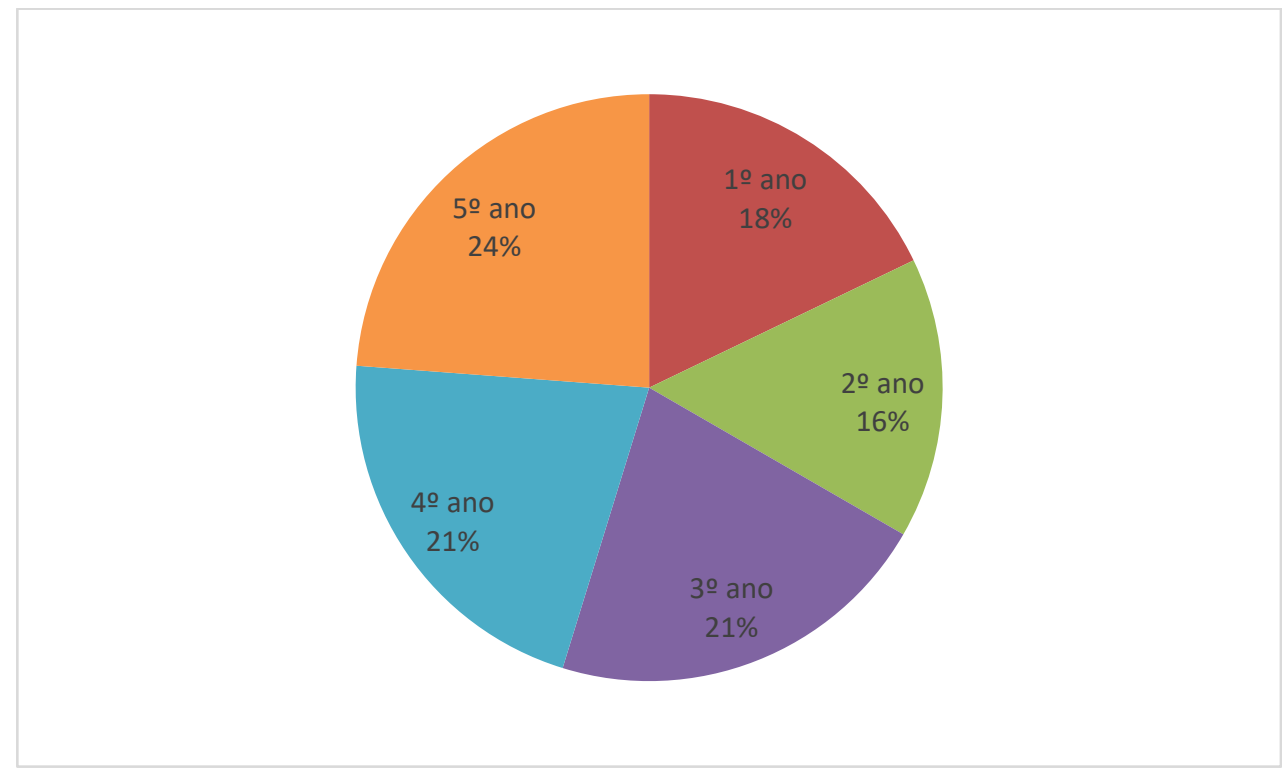

Figura 2.3. Totalidade de artigos organizados por ano escolar.

Sobre as áreas do conhecimento, foram localizadas publicações com abordagens pedagógicas em Linguagem (língua materna e segundo idioma), Matemática, Ciências da Natureza, Ciências Humanas e Arte, distribuídas conforme a figura 2.4. A catalogação dos artigos por área do conhecimento seguiu as diretrizes da Base Nacional Comum Curricular (Brasil, 2017), que estabelece cinco principais áreas do conhecimento. Além dos artigos catalogados por áreas do conhecimento, obteve-se dois artigos que tratam especificamente dos aspectos de engajamento, motivação e desafios com o uso da RA. 


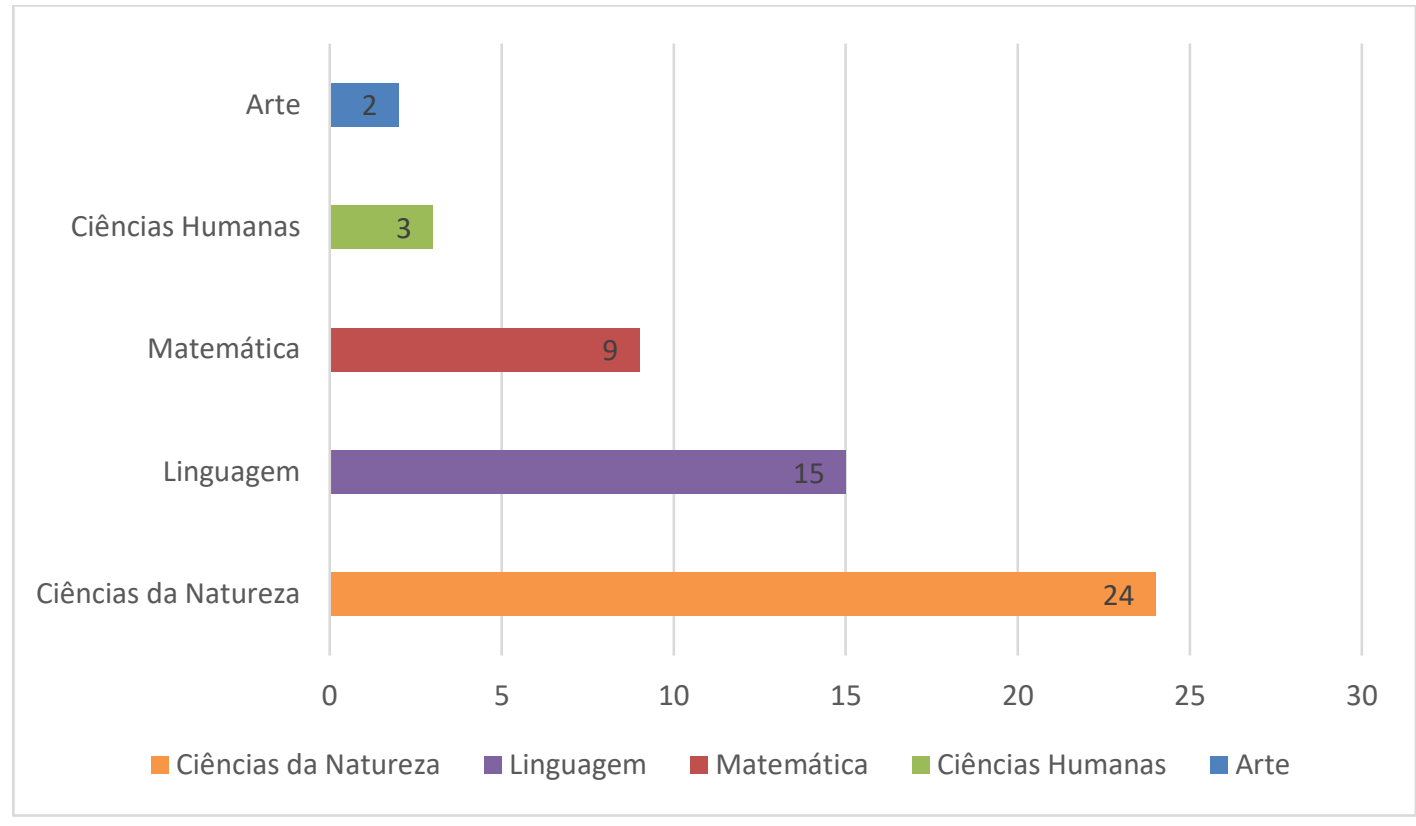

Figura 2.4. Publicações por área do conhecimento.

\section{Discussão}

A RA vem se consolidando como uma tecnologia de grande importância para a educação. Ao observar os anos em que ocorrem as publicações de artigos científicos envolvendo o uso da RA no ensino fundamental (anos iniciais) da educação básica, percebe-se o interesse crescente a partir do ano de 2012, principalmente. Nesse ano, houve o lançamento do primeiro aplicativo de RA em nuvem (Blippar, 2012) facilitando e aprimorando a tecnologia. No ano de 2016, ocorreu o lançamento do primeiro jogo de RA de grande sucesso: Pokémon Go. Com a disseminação da RA através dos jogos e o barateamento dos dispositivos, a partir do ano de 2016 há um maior número de publicações que envolvem educação e RA. Tais dados corroboram para a afirmativa de Tori e Hounsell (2018) de que a maior disseminação tecnológica e o barateamento dos dispositivos são fatores essenciais para a disseminação e consolidação do uso da RA em diversos contextos.

A universalização do acesso às tecnologias na educação, em especial a RA, é influenciada por diversos contextos e fatores em cada país. Encontra-se um grande número de artigos que tratam do uso da RA na educação, principalmente no continente asiático (31). Dados do Programa de Avaliação Internacional de Alunos (PISA) ${ }^{7}(\mathrm{OCDE}, 2019)$ identificam que os sistemas escolares asiáticos possuem os melhores resultados no desempenho dos alunos em Matemática, Ciências e Leitura. No que diz respeito à América do Sul, são localizados artigos apenas no Brasil (7) e no Equador (1), podendo indicar pouco investimento em pesquisa na área de educação e tecnologia no continente.

A RA possui grande potencial para enriquecer os processos educativos. O maior investimento em pesquisas que abordam RA está combinado aos tópicos de Ciências da Natureza no ensino fundamental (anos iniciais) da educação básica. Para catalogação e organização dos artigos analisados, foram utilizadas as três unidades temáticas de Ciências da Natureza da Base Nacional Comum Curricular (Brasil, 2017). São elas: Matéria e Energia; Terra e Universo e Vida e Evolução.

\footnotetext{
${ }^{7}$ https://www.oecd.org/pisa/
} 
"A unidade temática Matéria e Energia contempla o estudo de materiais e suas transformações, fontes e tipos de energia utilizados na vida em geral" (Brasil, 2017, p. 325). Gotardo et al. (2013) demonstram a utilização da RA para o ensino da coleta seletiva. O ciclo hidrológico é contemplado no estudo de Bratitsis, Bardanika e Ioannou (2017).

"Na unidade temática Terra e Universo, busca-se a compreensão de características da Terra, do Sol, da Lua e de outros corpos celestes" (Brasil, 2017, p. 328). Toledo-Morales e SanchesGarcia (2018) abordam os tópicos de representação da Terra, enquanto Syawaludin, Gunarhadi e Rintayati (2019) apresentam o uso da RA no estudo de rochas e Wickramapala (2019) aborda estudos ambientais. Phon et al. (2019) tratam dos aspectos da Astronomia.

Sobre Vida e Evolução, encontram-se "questões relacionadas aos seres vivos (incluindo os seres humanos)" (Brasil, 2017, p. 326). Três trabalhos tratam sobre a ecologia das borboletas: Tarng e Ou (2012); Hwang et al. (2016); e Bhagat et al. (2019). Sobre os animais, foram encontrados trabalhos que abordam a classificação da cadeia alimentar (Savitri, Aris, \& Supianto, 2019), as classes de animais (Flauzino \& Kirner, 2013), a conservação das tartarugas de Sumatra (Winarni \& Purwandari, 2019), os dinossauros (Lu et al., 2020) e os seres vivos em geral (Hendajani et al., 2019) (Chiang, Yang, \& Hwang, 2014). As plantas são contempladas nos artigos de Chang, Chung e Huang (2014), Maijarern, Chaiwut e Nobnop (2018), e Chen et al. (2020). O estudo do ser humano é retratado nos trabalhos de Hendajani et al. (2018), Kouzi, Mao e Zambrano (2019), Fakhrudin, Yamtinah e Riyadi (2017), Pérez-López e Contero (2013), e PérezLópez, Contero e Alcañiz (2010).

Del Río Guerra et al. (2020) demonstram em seu estudo que há melhora na pontuação média dos alunos na alfabetização científica utilizando RA móvel baseada em STEM (Science, Technology, Engineering and Mathematics) em comparação com o aprendizado convencional nos aspectos de contexto, conteúdo, competência e atitude científica dos alunos.

A análise realizada neste artigo demonstra que os tópicos da área de Ciências da Natureza possuem um número significativo de publicações (24), destacando-se dentre elas a unidade temática que aborda os seres vivos e todos os conteúdos que se desdobram a partir deste tema genérico (17). Tal análise sustenta a afirmação de que um dos aspectos relevantes do uso da RA na escola está na oferta da visualização e simulação de condições complexas de serem projetadas e acompanhadas no ambiente educativo. Exemplos positivos do uso da RA na área de Ciências da Natureza são a possibilidade de o aluno acompanhar a evolução e as fases de um ser vivo, ou ainda, compreender com profundidade sobre as tartarugas e borboletas, bem como aprender aspectos da Astronomia e da representação da Terra.

A área da Linguagem é fundamental em qualquer parte do mundo, pois os estudantes aprimoram suas experiências possibilitando a participação significativa e crítica nas diversas práticas sociais. Nessa área, os artigos localizados foram catalogados em: língua materna e segundo idioma.

Sobre a língua materna, foram localizados artigos que tratam da alfabetização no processo de grafia das palavras utilizando o recurso da RA (Silva, Silva, \& Melo, 2017), (Cavalcante et al., 2016) (Oliveira \& Zorzal, 2013). Os autores Silva, Silva e Melo (2017) apresentam o aplicativo Adoletras, que propõe auxiliar o processo de alfabetização de crianças na faixa etária entre seis e sete anos de idade. O Adoletras permite trabalhar a grafia das palavras e das sílabas com o uso de RA no celular. Segundo os autores, o uso desse dispositivo de RA possibilitou a aprendizagem significativa e a estimulação da imaginação das crianças. Cavalcante et al. (2016) demonstram a aplicação de RA em que o aluno, ao posicionar marcadores, faz a visualização da grafia da palavra associando-a à imagem do objeto sendo projetada, garantindo assim maior assimilação e reconhecimento da escrita da palavra. No estudo de Cavalcante et al. (2016) são utilizados os marcadores fiduciais. Esses marcadores geralmente possuem uma forma geométrica fixa e um 
identificador exclusivo, tem baixo custo e pouca manutenção, sendo amplamente utilizados. Entretanto, a RA também pode ser gerada por diversos tipos de imagem, reconhecimento do objeto ou ambiente, ou ainda por geolocalização, sensores inerciais ou por uma composição híbrida (Tori \& Hounsell, 2018).

Danaei et al. (2020) apresentam um trabalho direcionado à leitura de livros com e sem RA para alunos entre sete a nove anos de idade. Ainda, Carneiro e Silva (2016) utilizam o livro Banda da Floresta para a contação de histórias em RA, como recurso facilitador do processo de alfabetização.

Ao finalizar a etapa correspondente a alfabetização, a compreensão da leitura torna-se objeto de estudo, retratada no trabalho de Wangid, Rudyanto e Gunartati (2020) e voltada aos alunos dos $4^{\circ} \mathrm{s}$ e $5^{\circ} \mathrm{s}$ anos do ensino fundamental.

A aprendizagem de um segundo idioma requer a articulação entre esse e a língua materna. Camacho Montellanos, Macavilca Vásquez e Herrera Salazar (2019) apresentam um recurso de RA para a aprendizagem da língua quíchua pelos alunos da República da Nicarágua. O trabalho de Wen (2020) apresenta um jogo em RA para a composição de caracteres chineses. A aprendizagem da língua inglesa como segundo idioma ainda é amplamente difundida em diversas culturas e facilitada pelo uso de recursos de RA conforme os trabalhos de Chen et al. (2018), Chen e Wang (2015), Tsai (2018), Hsu (2017), Hsu (2019), Barreira et al. (2012), e Solak e Cakir (2015).

$\mathrm{Na}$ área de Matemática percebe-se a preocupação com o ensino e aprendizado dos conteúdos de Geometria. Os autores Purnama, Andrew e Galinium (2014), Rossano et al. (2020), Yu, Liao e Wu (2016), e Radu et al. (2015) abordam tópicos envolvendo o uso de RA para o ensino de geometria. No trabalho de Purnama, Andrew e Galinium (2014) pode-se conhecer uma ferramenta de auxílio para medir ângulos de 180 graus e também auxiliar o aluno no uso do transferidor para leitura de ângulos. Rossano et al. (2020) demonstram o aplicativo $\mathrm{Geo}^{+}$, que apoia a aprendizagem de geometria sólida. Yu, Liao e Wu (2016) desenvolveram ferramentas de RA para ajudar os alunos a criarem estratégias para calcular o volume de cubos. Radu et al. (2015) apresentam quebra-cabeças imersivos tridimensionais, ensinando Geometria e habilidades de cognição espacial. O trabalho de Lozada-Yánez, La-Serna-Palomino e Molina-Granja (2019) apresenta a aplicação de um sistema que envolve RA e sensor de movimento implementado na câmera MS-Kinect para o ensino de Matemática. O trabalho constata que os alunos tiveram atitudes positivas, destacando ser mais eficaz com alunos de baixo desempenho quando comparados àqueles de alto desempenho. Silva, Lemos e Carvalho (2014) apresentam um software para o apoio ao ensino de frações utilizando RA. Ao posicionar os marcadores, os alunos podem visualizar prismas geométricos que representam as frações em estudo. Radu, McCarthy e Kao (2016) investigaram aplicações educacionais de RA para Matemática. Foi realizada uma pesquisa com professores do ensino fundamental que elencaram os conteúdos de representação dos números, frações, problemas matemáticos e medição fora do padrão como difíceis de serem ensinados com métodos tradicionais. Foram criados protótipos que abordavam esses conteúdos e, ao serem aplicados com os estudantes, notaram-se ganhos significativos no processo de ensino e aprendizagem.

A área de Ciências Humanas contempla os conteúdos de História e Geografia. Foram identificados apenas três artigos nesta área que envolvem História: (Chang \& Lin, 2014), (Oltman \& Hammond, 2017) e (Casado, Gutiérrez, \& Somoza, 2018). Chang e Lin (2014) desenvolveram um sistema de aprendizagem baseado no Kinect para educar os alunos sobre os festivais culturais chineses, em especial o Festival de Lanternas. Oltman e Hammond (2017) relatam a experiência de aprendizagem através de um jogo sério que utilizou RA para o ensino da história da Morávia. Casado, Gutiérrez e Somoza (2018) tratam da integração da RA no ensino da unidade educacional que contempla as noções de tempo histórico. 
$\mathrm{Na}$ área das Artes, dois artigos abordam o ensino musical. Kusunoki, Sugimoto e Hashizume (2002) descrevem um sistema que apoia a aprendizagem musical de forma colaborativa. O estudo percebeu que os alunos ficaram mais interessados e curiosos para aprender sobre novos instrumentos musicais. Gomes et al. (2014) apresentam um jogo colaborativo baseado em geolocalização e RA que busca identificar compositores, instrumentos musicais e estilos, por meio de pistas envolvendo som, imagem, vídeo e gráficos.

Independentemente da etapa escolar em questão, um dos maiores desafios da educação é instigar a aprendizagem do aluno de forma enriquecedora, fazendo-o adquirir conhecimentos de forma significativa (Ausubel, 1963). Nos artigos analisados, que abordam a aquisição de conhecimento, identificou-se que o uso da RA pode melhorar este aspecto, quando comparado com abordagens tradicionais.

Syawaludin, Gunarhadi e Rintayat (2019) identificaram que o raciocínio abstrato dos alunos na aprendizagem de ciências aumentou em $24,20 \%$ ao utilizar multimídia interativa com base em RA. No estudo de Lozada-Yánez, La-Serna-Palomino e Molina-Granja (2019) há uma perspectiva que evidencia uma melhora na aprendizagem de $13 \%$ dos alunos com baixo desempenho em comparação ao grupo de alunos com alto desempenho, no qual a melhora concentrou-se em apenas 3,47\%, quando utilizado os recursos de RA. Pérez-Lópes e Contero (2013) obtiveram dados importantes ao analisar que há maior retenção na unidade de aprendizagem ensinada com RA em comparação ao que foi ensinado usando métodos tradicionais, quatro semanas após a avaliação inicial. Chiang, Yang e Hwang (2014) observaram em seu estudo que a RA permitiu que os alunos alcançassem fases mais elevadas no processo de construção do conhecimento.

Esses dados demonstraram que o uso da RA pode ter um impacto positivo em diversos contextos educacionais e que alunos que apresentam dificuldade na aprendizagem podem ser, ainda mais, beneficiados. Inclusive, é relatada maior retenção do conhecimento a longo prazo, garantindo que ocorreu a aprendizagem significativa do conteúdo proposto pelos educadores (Pérez-Lópes \& Contero, 2013).

Contudo, um artigo, ainda que isolado, não considera que a RA aumenta o nível de aprendizagem (Casado, Gutiérrez \& Somoza, 2018). Os autores compararam um grupo experimental e um de controle, verificando que a integração da RA não produz uma melhoria significativa na aprendizagem comparada ao uso de mídias tradicionais. Vale destacar que o estudo foi realizado em uma escola que já possui quadro branco digital e dispositivos móveis em sala de aula e são habituados a usá-los, pois as aulas são baseadas em livros digitais.

Educar é uma relação de construção e dialética que envolve diversos fatores e, entre eles, fatores emocionais. É antagônico falar de educação sem considerar o educando em sua totalidade. Stylianidou et al. (2020) tratam em seu trabalho especificamente sobre engajamento, participação e resposta à variabilidade dos alunos. Concluem que, ao utilizar a RA em contextos educativos, há aumento nos níveis de engajamento e participação na aprendizagem de todos os alunos, incluindo a variabilidade dos estudantes.

Durante a análise dos artigos selecionados, em apenas cinco não foram localizadas menções diretas sobre motivação, interesse, entusiasmo e interação entre os alunos. Dois trabalhos não consideram o uso de RA como motivador no processo educacional (Casado; Gutiérrez \& Somoza, 2018) e (Lu et al., 2020).

Casado, Gutiérrez e Somoza (2018) não identificaram melhora na motivação dos alunos, apenas uma melhora na sensação de participação pelos alunos. Lu et al. (2020) sugerem uma investigação mais aprofundada para determinar se o compartilhamento de dispositivos pode reduzir o engajamento e motivação. Em seus estudos, foi observado um declínio significativo na confiança dos alunos que estavam organizados em equipes de até seis pessoas e possuíam apenas 
um dispositivo. Assim, enquanto um aluno estava envolvido na montagem do quebra-cabeça, os outros apenas observavam.

Outro benefício do uso de RA na educação é relatado nos estudos de Wangid, Rudyanto e Gunartati (2020). Os autores estudaram os efeitos do uso de um livro em RA na ansiedade dos alunos na aprendizagem de Matemática. A ansiedade matemática pode ser uma barreira no processo de aprendizagem dos educandos e ao oferecer a visualização 3D por meio da RA, o estudo conclui que a mídia influencia positivamente e significativamente na ansiedade dos alunos.

Alalwan et al. (2020) realizaram entrevistas com 29 professores e identificaram sete problemas principais no que diz respeito ao uso de RA nos anos iniciais da educação básica. $\mathrm{O}$ maior desafio está relacionado à política BYOD ("Bring Your Own Device", em tradução livre: "traga seu próprio aparelho"). Apenas 7 dos 29 professores disseram que as famílias dos alunos não possuem condições de compra de aparelhos. A maior dificuldade do $B Y O D$ encontra-se na permissão das famílias para que o aluno traga seu equipamento para a escola, além da preocupação relacionada a roubos, perdas e uso indevido dos aparelhos. Outros problemas, além dos recursos serem limitados e da impossibilidade de adaptações por parte dos professores, estão relacionados, a longo prazo, a falta de: tempo, competência, atenção e de recursos. Isso evidencia que o fator novidade influi no uso dessa tecnologia, uma vez que o entusiasmo dos alunos ao utilizar a tecnologia pode gerar resultado positivo inicialmente.

Os alunos relatam que ficam menos entediados ao usar tecnologia nas aulas, o conteúdo apresenta-se de forma mais interessante e possibilita a aprendizagem de forma lúdica, mesmo quando não é apresentada em forma de jogo. Toledo-Morales \& Sanches-Garcia (2018) contribuem ao afirmarem que é necessário repensar as regras das escolas de ensino fundamental, especificamente dos anos iniciais, autorizando o uso dos dispositivos móveis.

Sobre os aspectos e recursos tecnológicos, apenas seis tratam do uso de RA sem uso de marcadores, são eles: sem marcadores (Hendajani et al., 2019), utilizando geolocalização (Gomes et al., 2014), (Chiang, Yang \& Hwang, 2014) e (Oltman \& Hammond, 2017) e o MS-Kinect (Lozada-Yánez, La-Serna-Palomino \& Molina-Granja, 2019) e (Chang \& Lin, 2014), que implica em uma adaptação da sala de aula para o uso desse dispositivo. A RA pode ser dividida em uso de marcadores e sem uso de marcadores. Os trabalhos mencionados, que não utilizam marcadores, fornecem informações virtuais com base na localização dos dispositivos através da função GPS.

Os artigos tratam do uso da RA com a dupla computador e webcam ou com o uso de aparelhos celulares e tablets, que podem ser fornecidos pela instituição ou permitido que os alunos tragam para a escola o seu aparelho de uso pessoal. Nos aspectos tecnológicos alguns problemas são relatados, como: dificuldade de acesso à internet, permissão das instituições para o download de aplicativos e falhas na leitura das aplicações de RA. Todos os estudos abordados implementaram suas aplicações, ainda que com focos de observação diferentes. Contudo, nenhum aplicativo foi encontrado para download e acesso, mesmo aqueles que pontuam essa possibilidade.

\section{Considerações Finais}

A partir de 2016, jogos de RA tornaram-se populares, atraindo atenção de diversos segmentos, entre eles, a educação. Os aspectos demográficos possibilitaram a percepção de uma tendência ao maior interesse da comunidade científica com a temática. $O$ continente asiático destaca-se no número de publicações, mas é possível verificar que o uso da RA nos contextos educativos está mundialmente difundido, e está presente em todos os anos escolares.

$\mathrm{O}$ uso da RA na educação pode possibilitar uma abordagem inovadora e a melhora na qualidade de ensino. O estudo relatado neste artigo forneceu argumentos que motivam a integração da RA no ensino fundamental (anos iniciais) da educação básica. Os resultados 
indicaram que pode haver melhora na aquisição do conhecimento, destacando-se inclusive os processos educativos dos alunos com baixo desempenho ou com variabilidade. A motivação e engajamento também foram estimulados na aprendizagem com o uso da RA. Alunos sentem-se atraídos pela tecnologia, motivados a aprender e engajados no seu processo educacional. Ainda que, alguns aspectos possam influir negativamente, como a baixa oferta de dispositivos e o consequente compartilhamento entre muitas pessoas e o fator novidade, as variáveis motivacionais são muito positivas. Também foi constatado que diversas áreas do conhecimento são beneficiadas com aplicativos de RA. Contudo, na área de Ciências Naturais há um maior investimento e interesse por parte dos pesquisadores e desenvolvedores de ferramentas de RA. Sugere-se que em estudos futuros se busque contemplar outras áreas do conhecimento com pouco investimento, como a área das Ciências Sociais, por exemplo.

Problemas e desafios são relatados para a efetivação do uso de realidade aumentada no ensino fundamental (anos iniciais) da educação básica. Há problemas técnicos, como oferta ao acesso à internet de qualidade, falhas na leitura das aplicações e disponibilidade de dispositivos de qualidade e em quantidade adequada. Outros são problemas institucionais, como as permissões para baixar aplicativos e a autorização aos alunos para que utilizem os dispositivos móveis. Há também os problemas sociais, como roubo e uso indevido de equipamentos. Ainda que observado o desejo de incorporar essa tecnologia nas suas aulas, os professores relatam receio pela falta de competência, treinamento e tempo. Os educadores também ressaltam o fato de os recursos serem limitados e da impossibilidade de adaptá-los aos contextos educacionais. Contudo, encontrar uma grande quantidade de trabalhos sendo desenvolvidos em várias partes do mundo, indica que, apesar dos problemas e dificuldades, a RA é uma tecnologia com grande potencial. Ao alinhar o uso da RA com uma metodologia educacional inovadora, propicia-se o sucesso no processo educativo e a aprendizagem significativa.

Por fim, essa revisão integrativa pode ser utilizada como instrumento norteador na organização de pesquisas futuras. Essas poderão contemplar áreas do conhecimento diferentes, aprofundar os estudos sobre aquisição do conhecimento e motivação com populações maiores, influência do fator novidade, buscar soluções relevantes às permissões de uso de dispositivos móveis por alunos de 6 a 11 anos de idade e criar aplicações de RA com outros equipamentos e tecnologias.

\section{Agradecimento}

Os autores agradecem ao curso de especialização em Computação Aplicada à Educação, do ICMC USP, Campus São Carlos, pelo apoio na realização deste trabalho.

$\mathrm{O}$ autor Romero Tori agradece ao $\mathrm{CNPq}$, pelo apoio recebido na forma de bolsa de produtividade em Desenvolvimento Tecnológico e Extensão Inovadora, na área de Tecnologias Sociais e Educacionais, processo 315180/2018-8.

\section{Referências}

Alalwan, N., Cheng, L., Al-Samarraie, H., Yousef, R., Alzahrani, A. I., \& Sarsam, S. M. (2020). Challenges and prospects of virtual reality and augmented reality utilization among primary school teachers: A developing country perspective. Studies in Educational Evaluation, 66, 100876. [GS Search]

Ausubel, D. P. (1963). The psychology of meaningful verbal learning. [GS Search] 
Azuma, R., Baillot, Y., Behringer, R., Feiner, S., Julier, S., \& MacIntyre, B. (2001). Recent advances in augmented reality. IEEE computer graphics and applications, 21(6), 34-47. doi: $\underline{10.1109 / 38.963459}$ [GS Search]

Bacca Acosta, J. L., Baldiris Navarro, S. M., Fabregat Gesa, R., \& Graf, S. (2014). Augmented reality trends in education: a systematic review of research and applications. Journal of Educational Technology and Society, 2014, 17(4), p. 133-149. [GS Search]

Bacich, L., \& Moran, J. (2018). Metodologias ativas para uma educação inovadora: uma abordagem teórico-prática. Penso Editora. [GS Search]

Barreira, J., Bessa, M., Pereira, L. C., Adão, T., Peres, E., \& Magalhães, L. (2012). MOW: Augmented Reality game to learn words in different languages: Case study: Learning English names of animals in elementary school. In 7th Iberian conference on information systems and technologies (CISTI 2012) (pp. 1-6). IEEE. [GS Search]

Bhagat, K. K., Liou, W. K., Michael Spector, J., \& Chang, C. Y. (2019). To use augmented reality or not in formative assessment: A comparative study. Interactive Learning Environments, 27(5-6), 830-840. doi: 10.1080/10494820.2018.1489857 [GS Search]

Bratitsis, T., Bardanika, P., \& Ioannou, M. (2017). Science education and augmented reality content: the case of the water circle. In 2017 IEEE 17th International Conference on Advanced Learning Technologies (ICALT) (pp. 485-489). IEEE. doi: 10.1109/ICALT.2017.64 [GS Search]

Brasil. (2017) Ministério da Educação. Secretaria da Educação Básica. Base Nacional Comum Curricular. Brasília, DF. Disponível em: http://basenacionalcomum.mec.gov.br/abase/

Montellanos, C., Luis, J., Vásquez, M., Alberto, C., \& Salazar, H. (2019). Augmented reality mobile application and its influence in Quechua language learning. In 2019 IEEE Sciences and Humanities International Research Conference (SHIRCON) (pp. 1-4). IEEE. doi: 10.1109/SHIRCON48091.2019.9024860 [GS Search]

Carneiro, R., \& da Silva, R. T. (2016). Banda da floresta. In Anais dos Workshops do Congresso Brasileiro de Informática na Educação (pp. 112-116). SBC. doi: 10.5753/cbie.wcbie.2016.112 [GS Search]

Casado, E. M., Cózar Gutiérrez, R., \& González-Calero Somoza, J. A. (2018). Incidencia de la Realidad Aumentada en la enseñanza de la historia: una experiencia en tercer curso de Educación Primaria. Incidencia de la Realidad Aumentada en la enseñanza de la historia: una experiencia en tercer curso de Educación Primaria, 23-39. [GS Search]

Cavalcante, R. S., Fernandes, F. G., Lamounier, E., \& Cardoso, A. (2016). Aplicação de realidade aumentada móvel para apoio ao ensino de crianças. In Anais dos Workshops do Congresso Brasileiro de Informática na Educação (pp. 691-700). SBC. doi: 10.5753/cbie.wcbie.2016.691 [GS Search]

Chang, R-C; Chung, L-Y \& Huang, Y-M. (2014). Developing an interactive augmented reality system as a complement to plant education and comparing its effectiveness with video learning. Interactive Learning Environments, 24(6), p. 1245-1264. doi:

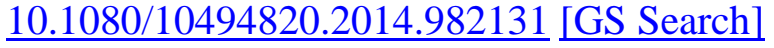

Chang, Y. H., \& Lin, Y. K. (2014). A kinect-based system for learning Chinese cultural festivals. In 2014 International Conference on Machine Learning and Cybernetics (Vol. 2, pp. 462468). IEEE.doi: 10.1109/ICMLC.2014.7009652 [GS Search]

Chen, C. P., \& Wang, C. H. (2015). The effects of learning style on mobile augmented-realityfacilitated English vocabulary learning. In 2015 2nd International Conference on Information 
Science and Security (ICISS) (pp. 1-4). IEEE. doi: 10.1109/ICISSEC.2015.7371036 [GS Search]

Chen, M. B., Wang, S. G., Chen, Y. N., Chen, X. F., \& Lin, Y. Z. (2020). A Preliminary Study of the Influence of Game Types on the Learning Interests of Primary School Students in Digital Games. Education Sciences, 10(4), 96. doi: 10.3390/educsci10040096 [GS Search]

Chen, S. Y., Hung, C. Y., Chang, Y. C., Lin, Y. S., \& Lai, Y. H. (2018). A study on integrating augmented reality technology and game-based learning model to improve motivation and effectiveness of learning English vocabulary. In 2018 1st International Cognitive Cities Conference (IC3) (pp. 24-27). IEEE. doi: 10.1109/IC3.2018.00015 [GS Search]

Chiang, T. H., Yang, S. J., \& Hwang, G. J. (2014). Students' online interactive patterns in augmented reality-based inquiry activities. Computers \& Education, 78, 97-108. doi: 10.1016/j.compedu.2014.05.006 [GS Search]

Danaei, D., Jamali, H. R., Mansourian, Y., \& Rastegarpour, H. (2020). Comparing reading comprehension between children reading augmented reality and print storybooks. Computers \& Education, 153, 103900.doi: 10.1016/j.compedu.2020.103900 [GS Search]

Danakorn Nincarean, A., Phon, L. E., Rahman, M. H. A., Utama, N. I., Ali, M. B., Abdi Halim, N. D., \& Kasim, S. (2019). The Effect of Augmented Reality on Spatial Visualization Ability of Elementary School Student. International Journal on Advanced Science Engineering Information Technology. Diakses dari https://core.ac.uk/download/pdf/296921451.pdf. [GS $\underline{\text { Searchl }}$

Del Río Guerra, M. S., Garza Martínez, A. E., Martin-Gutierrez, J., \& López-Chao, V. (2020). The Limited Effect of Graphic Elements in Video and Augmented Reality on Children's Listening Comprehension. Applied Sciences, 10(2), 527. doi: 10.3390/app10020527 [GS Search]

Fakhrudin, A., \& Yamtinah, S. (2017). Implementation of Augmented Reality Technology in Natural Sciences Learning of Elementary School to Optimize the Students' Learning Result. International Online Journal of Primary Education, 6(2), 30-38. [GS Search]

Flauzino, F. S., \& Kirner, C. (2013). Desenvolvimento de um Jogo Educacional sobre a Classificação dos Animais usando Realidade Aumentada Online. In Anais dos Workshops do Congresso Brasileiro de Informática na Educação(pp. 501-506). SBC. doi: 10.5753/cbie.wcbie.2013.501 [GS Search]

Ganong, L. H. (1987). Integrative reviews of nursing research. Research in nursing \& health, 10(1), 1-11. doi: 10.1002/nur.4770100103 [GS Search]

Gomes, J., Figueiredo, M., Gomes, C., \& Amante, L. (2014). Musical peddy-paper: a collaborative learning activity suported by augmented reality. In 11th International Conference on Cognition and Exploratory Learning in Digital Age. [GS Search]

Gotardo, R., De Groote, J. J., Volpini, N., Stamato, E., Dias, P., Almeida, T., \& Bueno, A. (2013). Realidade aumentada aliada aos materiais didáticos na educação básica. In Anais dos Workshops do Congresso Brasileiro de Informática na Educação (pp. 240-248). SBC. doi: 10.5753/cbie.wcbie.2013.240 [GS Search]

Hendajani, F., Hakim, A., Lusita, M. D., Saputra, G. E., \& Ramadhana, A. P. (2018). 3D animation model with augmented reality for natural science learning in elementary school. In Journal of Physics: Conference Series (Vol. 1013, No. 1, p. 012154). IOP Publishing. [GS $\underline{\text { Searchl }}$ 
Hendajani, F., Hakim, A., Sudiro, S. A., Saputra, G. E., \& Ramadhana, A. P. (2019). Tracking Visualization Of 3 Dimensional Object Natural Science Learning Media In Elementary School With Markerless Augmented Reality Based On Android. In Journal of Physics: Conference Series (Vol. 1192, No. 1, p. 012055). IOP Publishing. [GS Search]

Hsu, T. C. (2017). Learning English with augmented reality: Do learning styles matter?. Computers \& Education, 106, 137-149. doi: 10.1016/j.compedu.2016.12.007 [GS $\underline{\text { Search }}$

Hsu, T. C. (2019). Effects of gender and different augmented reality learning systems on English vocabulary learning of elementary school students. Universal Access in the Information Society, 18(2), 315-325.doi: 10.1007/s10209-017-0593-1 [GS Search]

Hwang, G. J., Wu, P. H., Chen, C. C., \& Tu, N. T. (2016). Effects of an augmented reality-based educational game on students' learning achievements and attitudes in real-world observations. Interactive Learning Environments, 24(8), 1895-1906. doi: $\underline{10.1080 / 10494820.2015 .1057747 \text { [GS Search] }}$

Kirner, C., \& Siscoutto, R. (2007, May). Realidade virtual e aumentada: conceitos, projeto e aplicações. In Livro do IX Symposium on Virtual and Augmented Reality, Petrópolis (RJ), Porto Alegre: SBC (Vol. 28). [GS Search]

El Kouzi, M., Mao, A., \& Zambrano, D. (2019). An educational augmented reality application for elementary school students focusing on the human skeletal system. In 2019 IEEE Conference on Virtual Reality and 3D User Interfaces (VR) (pp. 1594-1599). IEEE. doi: 10.1109/VR.2019.8798058 [GS Search]

Izaguirre, E. D. P., Abásolo, M. J., \& Collazos, C. A. (2020, October). Mobile Technology and Extended Reality for Deaf People: A systematic review of the open access literature. In 2020 XV Conferencia Latinoamericana de Tecnologias de Aprendizaje (LACLO) (pp. 1-8). IEEE. doi: 10.1109/LACLO50806.2020.9381186 [GS Search]

Khowaja, K., Banire, B., Al-Thani, D., Sqalli, M. T., Aqle, A., Shah, A., \& Salim, S. S. (2020). Augmented reality for learning of children and adolescents with autism spectrum disorder (ASD): A systematic review. IEEE Access, 8, 78779-78807. doi: 10.1109/ACCESS.2020.2986608 [GS Search]

Kusunoki, F., Sugimoto, M., \& Hashizume, H. (2002). Symphony-Q: A support system for learning music in collaborative learning. In IEEE International Conference on Systems, Man and Cybernetics (Vol. 4, pp. 6-pp). IEEE. doi: 10.1109/ICSMC.2002.1173254 [GS Search]

Lozada-Yánez, R., La-Serna-Palomino, N., \& Molina-Granja, F. (2019). Augmented Reality and MS-Kinect in the Learning of Basic Mathematics: KARMLS Case. International Education Studies, 12(9), 54-69. [GS Search]

Lu, S. J., Liu, Y. C., Chen, P. J., \& Hsieh, M. R. (2020). Evaluation of AR embedded physical puzzle game on students' learning achievement and motivation on elementary natural science. Interactive Learning Environments, 28(4), 451-463. doi: $\underline{10.1080 / 10494820.2018 .1541908 \text { [GS Search] }}$

Maijarern, T., Chaiwut, N., \& Nobnop, R. (2018). Augmented reality for science instructional media in primary school. In 2018 International Conference on Digital Arts, Media and Technology (ICDAMT) (pp. 198-201). IEEE. doi: 10.1109/ICDAMT.2018.8376523 [GS Search

Mendes, K. D. S., Silveira, R. C. D. C. P., \& Galvão, C. M. (2008). Revisão integrativa: método de pesquisa para a incorporação de evidências na saúde e na enfermagem. Texto \& contextoenfermagem, 17, 758-764. doi: 10.1590/S0104-07072008000400018 [GS Search] 
OCDE (2019), PISA 2018 Assessment and Analytical Framework, PISA, OECD Publishing, Paris. [GS Search]

OECD (2018). Education at a Glance 2018: OECD Indicators, OECD Publishing, Paris. [GS Search]

de Oliveira, B. M., \& Zorzal, E. R. (2013). Jogo de Realidade Aumentada para Auxiliar no Aprendizado Infantil. In Anais dos Workshops do Congresso Brasileiro de Informática na Educação (pp. 507-510). SBC. doi: 10.5753/cbie.wcbie.2013.507 [GS Search]

Oltmana, J. L., \& Hammondb, T. C. "I Almost Wanted to Touch It": Flow and Learning in GameBased History Education with Augmented Reality for Early Elementary Students. AERA Online Paper Repository. [GS Search]

Pellas, N., Fotaris, P., Kazanidis, I., \& Wells, D. (2019). Augmenting the learning experience in primary and secondary school education: A systematic review of recent trends in augmented reality game-based learning. Virtual Reality, 23(4), 329-346. doi: 10.1007/s10055-018-0347$\underline{2}$ [GS Search]

Pérez-López, D., \& Contero, M. (2013). Delivering educational multimedia contents through an augmented reality application: A case study on its impact on knowledge acquisition and retention. Turkish Online Journal of Educational Technology-TOJET, 12(4), 19-28. [GS Search]

Pérez-López, D., Contero, M., \& Alcaniz, M. (2010). Collaborative development of an augmented reality application for digestive and circulatory systems teaching. In 2010 10th IEEE International Conference on Advanced Learning Technologies (pp. 173-175). IEEE. doi: 10.1109/ICALT.2010.54 [GS Search]

Purnama, J., Andrew, D., \& Galinium, M. (2014). Geometry learning tool for elementary school using augmented reality. In 2014 International Conference on Industrial Automation, Information and Communications Technology (pp. 145-148). IEEE. doi: 10.1109/IAICT.2014.6922112 [GS Search]

Radu, I., McCarthy, B., \& Kao, Y. (2016). Discovering educational augmented reality math applications by prototyping with elementary-school teachers. In 2016 IEEE Virtual Reality (VR) (pp. 271-272). IEEE. doi: 10.1109/VR.2016.7504758 [GS Search]

Radu, I., Doherty, E., DiQuollo, K., McCarthy, B., \& Tiu, M. (2015). Cyberchase shape quest: pushing geometry education boundaries with augmented reality. In Proceedings of the 14th International Conference on Interaction Design and Children (pp. 430-433). doi: $\underline{10.1145 / 2771839.2771871 \text { [GS Search] }}$

Rossano, V., Lanzilotti, R., Cazzolla, A., \& Roselli, T. (2020). Augmented reality to support geometry learning. IEEE Access, 8, 107772-107780. doi: 10.1109/ACCESS.2020.3000990 [GS Search]

Savitri, N., Aris, M. W., \& Supianto, A. A. (2019). Augmented reality application for science education on animal classification. In 2019 International Conference on Sustainable Information Engineering and Technology (SIET)(pp. 270-275). IEEE. doi: 10.1109/SIET48054.2019.8986031 [GS Search]

Silva, T. R., Lemos, B. M., \& de Alencar Carvalho, C. V. (2014). UM SOFTWARE EDUCACIONAL PARA APOIO AO ENSINO DE FRAÇÕES UTILIZANDO REALIDADE AUMENTADA. Acta Scientiae et Technicae, 2(2). doi: 10.17648/uezo-astv2i2.44 [GS Search] 
Silva, T., da Silva, A., \& Melo, J. (2017). Adoletras: Um jogo de Realidade Aumentada para auxiliar no processo de Alfabetização. In Anais dos Workshops do Congresso Brasileiro de Informática na Educação (pp. 206-213). SBC. doi: 10.5753/cbie.wcbie.2017.206 [GS $\underline{\text { Searchl }}$

Sirakaya, M., \& Alsancak Sirakaya, D. (2018). Trends in Educational Augmented Reality Studies: A Systematic Review. Malaysian Online Journal of Educational Technology, 6(2), 60-74. [GS Search]

Solak, E., \& Cakir, R. (2015). Investigating the role of augmented reality technology in the language classroom. Online Submission, 18(4), 1067-1085. [GS Search]

van der Stappen, A., Liu, Y., Xu, J., Yu, X., Li, J., \& Van Der Spek, E. D. (2019). MathBuilder: A collaborative AR math game for elementary school students. In Extended Abstracts of the Annual Symposium on Computer-Human Interaction in Play Companion Extended Abstracts (pp. 731-738). doi: 10.1145/3341215.3356295 [GS Search]

Stylianidou, N., Sofianidis, A., Manoli, E., \& Meletiou-Mavrotheris, M. (2020). "Helping Nemo!"-Using Augmented Reality and Alternate Reality Games in the Context of Universal Design for Learning. Education Sciences, 10(4), 95. doi: 10.3390/educsci10040095 [GS $\underline{\text { Search }]}$

Syawaludin, A., Gunarhadi, G., \& Rintayati, P. (2019). Enhancing elementary school students' abstract reasoning in science learning through augmented reality-based interactive multimedia. Jurnal Pendidikan IPA Indonesia, 8(2), 288-297. doi: 10.15294/jpii.v8i2.19249 [GS Search]

TAJRA, S. F. (2019). Informática na educação: o uso de tecnologias digitais na aplicação das metodologias ativas. São Paulo: Érica. [GS Search]

Tarng, W., \& Ou, K. L. (2012). A study of campus butterfly ecology learning system based on augmented reality and mobile learning. In 2012 IEEE seventh international conference on wireless, mobile and ubiquitous technology in education (pp. 62-66). IEEE. doi: 10.1109/WMUTE.2012.17 [GS Search]

Toledo-Morales, P., \& Sanchez-Garcia, J. M. (2018). Use of augmented reality in social sciences as educational resource. Turkish Online Journal of Distance Education, 19(3), 38-52. doi: 10.17718/tojde.444635 [GS Search]

Tori, R. (2018). Educação sem distância: as tecnologias interativas na redução de distâncias em ensino e aprendizagem (Vol. 9). Artesanato Educacional LTDA. [GS Search]

Tori, R., \& da Silva Hounsell, M. (2018). Introdução a realidade virtual e aumentada. Interação, 7 , 11. [GS Search]

Tsai, C. C. (2018). A Comparison of EFL Elementary School Learners' Vocabulary Efficiency by Using Flashcards and Augmented Reality in Taiwan. Stanisław Juszczyk, 53. doi: 10.15804/tner.2018.51.1.04 [GS Search]

Wahyu, Y., Suastra, I. W., Sadia, I. W., \& Suarni, N. K. (2020). The Effectiveness of Mobile Augmented Reality Assisted Stem-Based Learning on Scientific Literacy and Students' Achievement. International Journal of Instruction, 13(3), 343-356. [GS Search]

Wangid, M., Rudyanto, H., \& Gunartati, G. (2020). The Use of AR-Assisted Storybook to Reduce Mathematical Anxiety on Elementary School Students. [GS Search]

Wen, Y. (2020). An Augmented Paper Game with Socio-Cognitive Support. IEEE Transactions on Learning Technologies, 13(2), 259-268. doi: 10.1109/TLT.2019.2924216 [GS Search] 
Wickramapala, T., Jayawardhana, L., Tharaki, S., Senevirathna, S., Gamage, N., \& Wickramarathna, J. (2019). Easy Learning: Augmented Reality Based Environmental Studies for Primary Students. In 2019 International Conference on Advancements in Computing (ICAC) (pp. 255-260). IEEE. doi: 10.1109/ICAC49085.2019.9103402 [GS Search]

Winarni, E. W., \& Purwandari, E. P. (2019). The Effectiveness of Turtle Mobile Learning Application for Scientific Literacy in Elementary School. Journal of Education and eLearning Research, 6(4), 156-161. [GS Search]

Yu, C. H., Liao, Y. T., \& Wu, C. C. (2016). Using augmented reality to learn the enumeration strategies of cubes. In 2016 IEEE International Conference on Teaching, Assessment, and Learning for Engineering (TALE) (pp. 412-418). IEEE. doi: 10.1109/TALE.2016.7851832 [GS Search] 